\title{
A Graph-based Dataset of Commit History of Real-World Android apps
}

\author{
Franz-Xaver Geiger \\ Vrije Universiteit Amsterdam \\ The Netherlands \\ f.geiger@student.vu.nl \\ Fabio Palomba \\ University of Zurich \\ Switzerland \\ palomba@ifi.uzh.ch
}

\author{
Ivano Malavolta \\ Vrije Universiteit Amsterdam \\ The Netherlands \\ i.malavolta@vu.nl \\ Dario Di Nucci \\ Vrije Universiteit Brussel \\ Belgium \\ ddinucci@vub.ac.be
}

\author{
Luca Pascarella \\ Delft University of Technology \\ The Netherlands \\ l.pascarella@tudelft.nl \\ Alberto Bacchelli
University of Zurich
Switzerland
bacchelli@ifi.uzh.ch
}

\begin{abstract}
Obtaining a good dataset to conduct empirical studies on the engineering of Android apps is an open challenge. To start tackling this challenge, we present AndroidTimeMachine, the first, self-contained, publicly available dataset weaving spread-out data sources about real-world, open-source Android apps. Encoded as a graph-based database, AndroidTimeMachine concerns 8,431 real open-source Android apps and contains: (i) metadata about the apps' GitHub projects, (ii) Git repositories with full commit history and (iii) metadata extracted from the Google PlAY store, such as app ratings and permissions.
\end{abstract}

\section{CCS CONCEPTS}

\section{- Software and its engineering $\rightarrow$ Maintaining software;}

\section{KEYWORDS}

Android, Mining Software Repositories, Dataset

\section{INTRODUCTION}

Since mobile apps differ from traditional software and require to tackle new problems (e.g., power management and privacy protection $[5,7,15,16])$, researchers are conducting empirical studiesespecially by mining software repositories-to understand and support mobile software development.

As an example of recent research on apps, Malavolta et al. analyzed more than 11,000 apps published in the Google Play store and investigated the end users' perceptions about various hybrid development frameworks [12]. Also, Linares-Vásquez et al. mined 54 Android apps from the Google Play store to find programming practices that may lead to an excessive energy consumption [5].

Permission to make digital or hard copies of all or part of this work for personal or classroom use is granted without fee provided that copies are not made or distributed for profit or commercial advantage and that copies bear this notice and the full citation on the first page. Copyrights for components of this work owned by others than the author(s) must be honored. Abstracting with credit is permitted. To copy otherwise, or republish, to post on servers or to redistribute to lists, requires prior specific permission and/or a fee. Request permissions from permissions@acm.org.

MSR '18, May 28-29, 2018, Gothenburg, Sweden

() 2018 Copyright held by the owner/author(s). Publication rights licensed to Association for Computing Machinery.

ACM ISBN 978-1-4503-5716-6/18/05 ..\$15.00

https://doi.org/10.1145/3196398.3196460
A common challenge when investigating apps is accessing candidate subjects (i.e., the app binaries or source code). A widely adopted approach is to gather information from open-source software (OSS) market places, F-Droid ${ }^{1}[4,9,13]$. Nevertheless, relying on F-Droid impacts the number of projects that can be considered, as it only contains metadata of 2,697 apps. $^{2}$ Moreover, for every study, researchers have to (i) systematically explore several online repositories to find analyzable apps, (ii) filter out source code not intended for the Android platform, and (iii) verify apps' consistency within official distribution channels.

To improve this situation, we propose AndroidTimeMachine, a graph-based dataset with data linked from different sources concerning the development and publication process of 8,431 OSS Android apps. We combine information from GitHub and Google Play to create a unified dataset including (i) metadata of GITHuB projects, (ii) full commit and code history, and (iii) metadata from the Google Play store. This dataset is the largest collection of published OSS Android apps with linked source code and store meta-data that we know of. The connected nature of this dataset and the included revision history allow a holistic view on OSS Android apps from development to publication on Google Play.

AndroidTimeMachine is composed of two main parts: A graphbased database (which facilitates understanding and navigation by focusing on links between apps, repositories, commits, and contributors) and a GiT server hosting a mirror of all 8,431 GitHuB repositories (thus providing a self-contained snapshot of the apps within the dataset). AndroidTimeMachine is publicly accessible at http://androidtimemachine.github.io and it is available as a Docker container image, which runs an instance of a NEO4J database with all the metadata and a GitLAB server hosting all the mirrored GitHub repositories.

\section{DATASET}

Creating AndroidTimeMachine involved retrieving large quantities of information from several sources and combining it by linking it based on available identifiers. During this process we had to deal with limitations on how these sources select and publish data and how they restrict access, e.g., through rate limits. We detail the

\footnotetext{
${ }^{1}$ https://f-droid.org/en/

${ }^{2}$ References counted on March 12, 2018 from https://gitlab.com/fdroid/fdroiddata/ tree/747a2662f82665b66c70cbcee5520068282d20ee/metadata
} 
process we used to identify the Android apps in our dataset (Section 2.1), the structure of our Neo4j database (Section 2.2), and the distribution of our dataset (Section 2.3). Furthermore, we showcase how the data can be used (Section 2.4) and point out limitations in Section 2.5 .

\subsection{Apps Identification}

To create our dataset we defined a 4-step process (see Figure 1), which: (1) identifies open-source Android apps hosted on GiтHuB, (2) extracts their package names, (3) checks their availability on the Google Play store, and (4) matches each GitHub repository to its corresponding app entry in the Google Play store. ${ }^{3}$
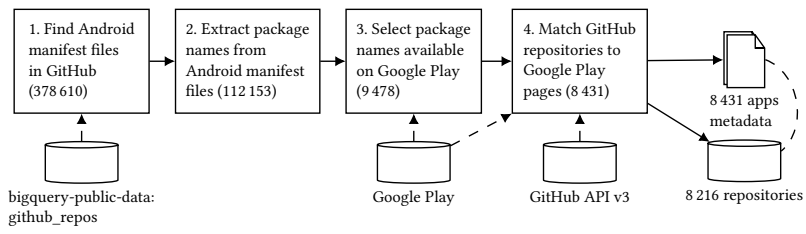

Figure 1: App Identification Process

Step 1. Identification of Android manifest files in GitHub. Step 1 aims at finding all repositories on GiтHuв potentially containing the source code of an Android app. Since each Android app is required to contain an XML file named AndroidMani fest. xml (which describes the app metadata and how it interacts with the Android system [11]), we performed this step by searching for AndroidManifest.xml files across all repositories on GiтHub. Our search has been performed on the publicly-available GiтHuв mirror available in BIGQUERY. ${ }^{4}$ This mirror contains information about files in all open-source repositories on GiтHuB, making it a good interface for finding repositories containing certain file types [3]. Our query returned 378,610 AndroidManifest.xml files across 124,068 repositories (search performed in October 2017).

Step 2. Extraction of Android package names. Repositories may contain more than one manifest file, e.g., when they host the code of more than one app (e.g., free and paid versions) or include third-party code (e.g., libraries with their own manifest file) This complicates matching repositories to apps and warrants the heuristic algorithm in step 4. In every AndroidMani fest.xml file, the root element must also include a package attribute containing the unique identifier of the app in the Google PlAY store. In this step we queried the BIGQUERY table containing the raw contents of all AndroidManifest.xml files and extracted the package names of their corresponding apps. The result of this query was a collection of 112,153 package names. This step still contained duplicated package names, mainly due to frequent usage of common names for test or toy projects, inclusion of libraries, or because repositories got forked [8]; this was taken care of in the following step(s).

Step 3. Selection of package names in Google Play. In this step we aimed at excluding all test, library, or toy projects. By using the package name as app identifier, we filtered out all those apps for which there was no corresponding webpage in the Google PlaY

\footnotetext{
${ }^{3}$ Code and queries at: https://github.com/androidtimemachine/open_source_ android_apps

${ }^{4}$ https://cloud.google.com/bigquery/public-data/github
}

store. This filtering step excluded all unpublished and non-existent package names, leading to 9,478 potentially-real app identifiers. Metadata for these apps was downloaded from the app store using a publicly available web scraper called node-google-play. ${ }^{5}$

Step 4. App-repository matching. In this step, Google Play pages got mapped to GiтHuв repositories, via heuristics. We linked a package name to a repository if the repository was the only one containing an AndroidManifest. xml file for a given package name $(77.1 \%)$. If more than one repository existed with the same package name, we searched metadata of the Google Play entry for mentions of GitHuB repository URLs. We matched a repository to the package name if we found links to exactly one repository (6.6\%). Finally, in cases in which neither of the two previous approaches resulted in a match, we selected the most popular repository based on number of (i) forks, (ii) watchers, and (iii) subscribers (5.0\%). We discarded 1,047 package names for which we could not determine a unique match or which were not accessible on GiтHub anymore.

These four steps resulted in a collection of 8,431 real Android apps whose source code is available in 8,216 GitHuB repositories.

\subsection{Database Structure}

To make data about OSS Android applications easily accessible and queryable, we designed and populated a graph-based database representing all the data gathered during the app identification process and the metadata related to each GiтHuB commit within the dataset (e.g., number of changes and contributors). The database is persisted using Neo4j (i.e., a graph $\mathrm{DBMS}^{6}$ ), thus researchers can use algorithms from graph theory for investigations (e.g., reconstructing the chain of commits across the whole lifetime of the app and identifying apps in a certain category with at least $n$ active developers in a certain timeframe); morever, our dataset can be accessed: (i) with Cypher, a domain-specific graph query language, (ii) via a native Java API, and (iii) via a dedicated HTTP REST API.

Figure 2 shows the structure of the database. Data points are stored as nodes connected by relationships (i.e., the edges of the graph); both nodes and edges can have properties.

Node types and their properties. Android apps are represented as nodes of type App. They include the package name used to identify the app as string property id. The node type GooglePlayPage holds the metadata we mined from the Google PlAy entry of the app, such as its title, package name, average rating, and requested permissions. The Gi tHubRepository node represents a GiTHuB project with its id (i.e., the fixed internal identifier for repositories on GITHuB). All other properties of GitHubReposi tory nodes represent a subset of data accessible through GitHuB API v3, such as the owner, forks count, and repository name. A Commit node describes a commit of the GIT repository. The id property is the full hash of the commit. The node also contains short_id, number of changed lines (additions, deletions, total), as well as the commit title and message. Both authors and committers are represented by the node type Contributor. This node type has an email and a name property. Contributor nodes get merged by email, i.e., only the latest name seen during creation of the database is accessible. They can be differentiated by their relationship to a

\footnotetext{
${ }^{5}$ https://github.com/dweinstein/node-google-play-cli

${ }^{6}$ http://neo4j.com
} 


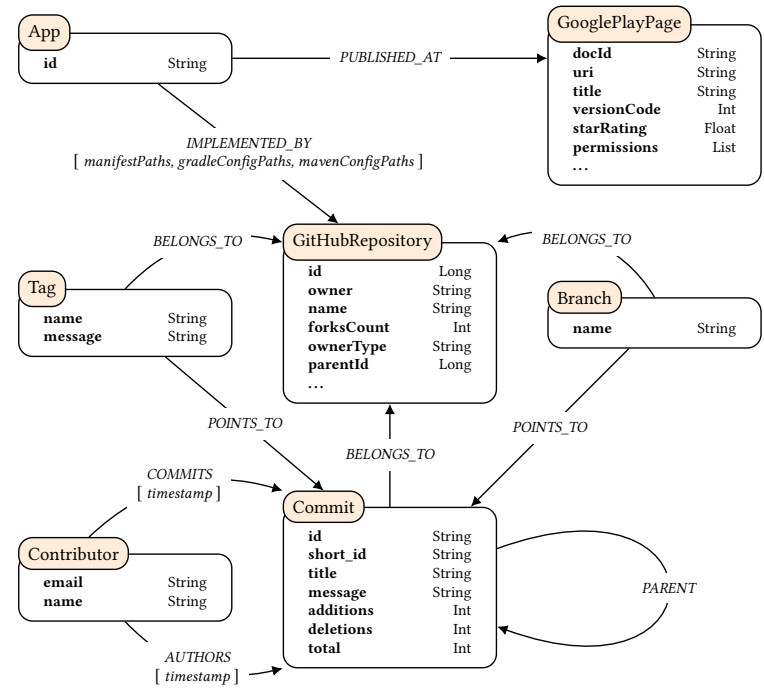

Figure 2: Schema of our dataset, as persisted in Neo4j (a graph database management system).

Commit. Finally, Git tags and branches are stored as nodes of type Tag and Branch, respectively. Both node types have a property name. Tags may also include the message stored with the tag.

Relationships between nodes. Relationships are directed graph edges between nodes and can contain properties. PUBLISHED_AT relations connect App nodes to their corresponding Google PlAY node. The link between an app and its corresponding GiтHuB repository (IMPLEMENTED_BY) contains the following properties: the paths to its Android manifest files (manifestPaths) and the paths to its build configuration files (gradleConfigPaths or mavenConfigPaths). Branches, tags, and commits are linked to a GITHuB repository with edges of type BELONGS_TO. A POINTS_TO relation connects Branch and Tag nodes to a Commit. Version control history between commits is represented with the PARENT relation, which is a many-to-many relation due to the nature of branches and merges of GIT. The COMMITS and AUTHORS relationship indicate the Contributor who authored and committed a change. Both relationships store a timestamp of their event.

\subsection{Dataset Availability}

As explained in Section 2, our dataset is composed of: (i) a Neo4j graph database with metadata of identified apps and (ii) a list of GiтHuB repositories. For ease of use and reproducibility, we make available a Docker-based containerized version of the entire data with pre-installed software necessary to show, explore, and query the data. Docker containers are a good way of sharing runnable environments with all dependencies included [2].

The total size of all GiT repositories in the dataset is 136GB. Since not all researchers may need to access the full dataset, we split the data into two containers, where one Docker image contains the Neo4j database ${ }^{7}$ and the second container serves as a snapshot of

\footnotetext{
${ }^{7}$ https://hub.docker.com/r/androidtimemachine/neo4j_open_source_android_
}

all GitHub repositories in the dataset cloned to a local Gitlab. ${ }^{8}$ All information from the graph database is also available in CSV format in the GiT repository of the docker image. ${ }^{9}$

\subsection{Dataset Usage}

Researchers can access our dataset through the Neo4j and GitlaB web interfaces, as well as through their respective REST-based APIs. The GitLAB web server and its API are accessible on port $80,{ }^{10}$ while the Neo4j instance can be accessed through default ports 7474 for the HTTP protocol and port 7687 for the BOLT protocol used for Cypher queries. ${ }^{11}$ In the Neo4j database, the snapshot attribute of GitHubRepository nodes links to the address of the corresponding repository in our GitLab instance. Documentation on how to run the container and access the data is in the Docker image repository. ${ }^{7}$

The connected nature of the graph database facilitates many potential research questions. In the following we showcase queries and analyses supported by our dataset. ${ }^{12}$

Scenario 1. Select apps belonging to the Finance category with more than 10 commits in a given week.

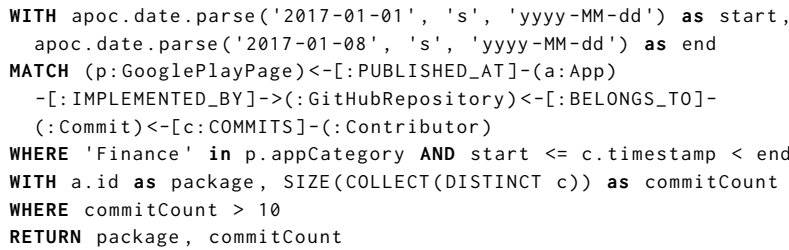

Scenario 2. Select contributors who worked on more than one app in a given year.

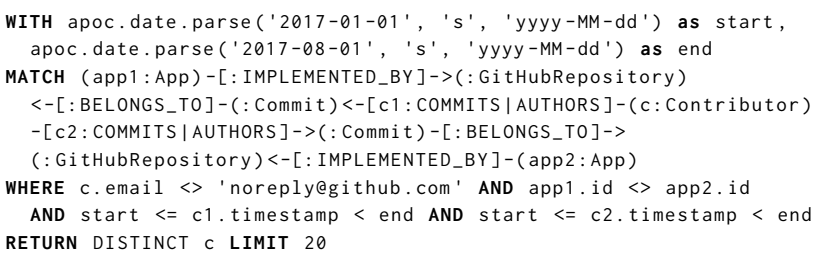

Scenario 3. Providing our dataset in containerized form allows future research to easily augment the data and combine it for new insights. The following is a very simple example showcasing this possibility. Assuming all commits have been tagged with self-reported activity of developers, select all commits in which the developer is fixing a performance bug. We apply a simple tagger, but a more advanced model (e.g., [14]) would lead to better results. MATCH (c:Commit) WHERE c.message CONTAINS 'performance'

SET C :PerformanceFix

Also, given these additional labels, performance related fixes can then be used in any kind of query via the following snippet.

MATCH (c: Commit:Performancefix) RETURN C LIMIT 20

\footnotetext{
${ }^{8}$ https://androidtimemachine.github.io/dockerImages

${ }^{9}$ https://github.com/AndroidTimeMachine/neo4j_open_source_android_apps/ tree/master/data

${ }^{10}$ Username: root - password: gitlab. Documentation of the GITLAB API is available in the container at endpoint /help/api/README.md and a potentially newer version at https://docs.gitlab.com/ce/api/

${ }^{11} \mathrm{Neo} 4 \mathrm{j}$ documentation available at https://neo4j.com/graphacademy/

${ }^{12}$ Some of the examples rely on the Neo4j plugin APOC, which can be installed by mapping an external directory into the Docker image: https://guides.neo4j.com/apoc
} 
Scenario 4. Metadata from GitHub and Google Play can be combined and compared. Both platforms have popularity measures, e.g., star ratings, which are returned by the following query.

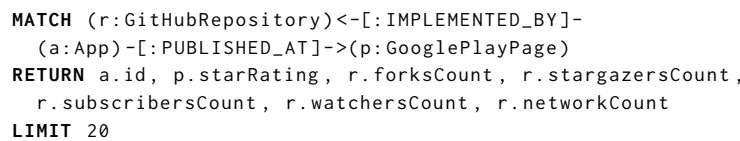

Scenario 5. Is a higher number of contributors related to the success of an app? The following query returns the average rating on Google Play and the number of contributors to the code by app.

MATCH (c: Contributor)-[: AUTHORS| COMMITS ]->(: Commit)

$-[:$ BELONGS_TO $]->(:$ GitHubRepository $)<-[:$ IMPLEMENTED_BY $]-$

(a: App) -[: PUBLISHED_AT ]->(p: GooglePlayPage)

WITH p.starRating as rating, a.id as package,

SIZE (COLLECT (DISTINCT C)) as contribcount

RETURN package, rating, contribcount LIMIT 20

\subsection{Dataset Limitations}

We only considered applications available in the Google Play store. This limitation is mitigated by the fact that Google PlaY is the official Android app store and offers the largest selection of Android apps [1]. We mined Google Play from a server in our region, thus limiting the data collection to the apps available here.

Data selection can be biased by the presence of the source code on GiтHuв. We consider this acceptable considering that, in the recent years, GiтHuв has been the most known platform for the open-source community and it offers a large and diverse selection of OSS projects [6].

Searching candidate repositories using the GitHub API was not possible due to limitations on the number of results returned by each query. Indeed, even when stratifying search queries (e.g., by filesize, with a byte-level granularity), not all the results could be retrieved. We overcame this issue by using BIGQUERY.

Resorting to a heuristic approach for matching Google PlaY listings to GITHUB repositories entails the risk of mismatches. Especially the $5.0 \%$ of apps that were linked by popularity measures might have been wrongly classified. However, confidence of correct matches is high for the $77.1 \%$ of apps for which only a unique repository contains an AndroidManifest.xml file.

\section{RELATED WORK}

Previous studies created data collections of OSS Android applications. For their study on app releases, Nayebi et al. [13] linked 69 F-Droid apps with version control repositories. Where available, metadata from Google PLAY was included. A similar dataset of OSS Android apps was constructed by Krutz et al. [9] to facilitate security research [10]. Das et al. [4] used F-Droid as a starting point for identifying open-source Android apps. They built a dataset for the analysis of performance related commits of mobile applications by matching apps listed on F-Droid against GiтHuB repositories. Later, the apps were filtered considering their availability on GoogLE Play. The final dataset was composed of 2,443 apps.

These datasets have the advantage that F-Droid contains executable app packages which our collection does not include. However, AndroidTimeMachine covers more apps than listed on F-Droid because we identify candidate repositories searching the Android app manifest; this approach provides a more realistic sample of open-source Android apps and increase the number and diversity of apps to perform research on.

\section{CONCLUSIONS}

We created AndroidTimeMachine, a dataset of 8,431 real-world open-source Android apps. It combines source and commit history information available on GiтHuB with the metadata from GoogLE PLAY store. The graph representation used for structuring the data eases the analysis of the relationships between source code and metadata. The dataset is provided as Docker container to improve its accessibility and extensibility.

\section{ACKNOWLEDGMENTS}

Bacchelli and Palomba gratefully acknowledge the support of the Swiss National Science Foundation through the SNF Project No. PP00P2_170529. Di Nucci is funded by the Belgian Innoviris TeamUp project INTiMALS.

\section{REFERENCES}

[1] Ben Martin. 2017. The Global Mobile Report - comScore's cross-market comparison of mobile trends and behaviours. (2017). ComsCore white paper.

[2] Ryan Chamberlain and Jennifer Schommer. 2014. Using Docker to support reproducible research. DOI: https://doi.org/10.6084/m9.figshare 1101910 (2014).

[3] Jürgen Cito, Gerald Schermann, John Erik Wittern, Philipp Leitner, Sali Zumberi, and Harald C. Gall. 2017. An empirical analysis of the Docker container ecosystem on GitHub. In Proceedings of the 14th International Conference on Mining Software Repositories. IEEE Press, 323-333.

[4] Teerath Das, Massimiliano Di Penta, and Ivano Malavolta. 2016. A Quantitative and Qualitative Investigation of Performance-Related Commits in Android Apps. In 2016 IEEE International Conference on Software Maintenance and Evolution, ICSME 2016, Raleigh, NC, USA, October 2-7, 2016. 443-447.

[5] Dario Di Nucci, Fabio Palomba, Antonio Prota, Annibale Panichella, Andy Zaidman, and Andrea De Lucia. 2017. Software-based energy profiling of android apps: Simple, efficient and reliable?. In Software Analysis, Evolution and Reengineering (SANER), 2017 IEEE 24th International Conference on. IEEE, 103-114.

[6] Georgios Gousios and Diomidis Spinellis. 2017. Mining software engineering data from GitHub. In Proceedings of the 39th International Conference on Software Engineering Companion. IEEE Press, 501-502.

[7] Mona Erfani Joorabchi, Ali Mesbah, and Philippe Kruchten. 2013. Real challenges in mobile app development. In Empirical Software Engineering and Measurement, 2013 ACM/IEEE International Symposium on. IEEE, 15-24.

[8] Eirini Kalliamvakou, Georgios Gousios, Kelly Blincoe, Leif Singer, Daniel M German, and Daniela Damian. 2016. An in-depth study of the promises and perils of mining GitHub. Empirical Software Engineering 21, 5 (2016), 2035-2071.

[9] Daniel E. Krutz, Mehdi Mirakhorli, Samuel A. Malachowsky, Andres Ruiz, Jacob Peterson, Andrew Filipski, and Jared Smith. 2015. A dataset of open-source Android applications. In Proceedings of the 12th Working Conference on Mining Software Repositories. IEEE Press, 522-525.

[10] Daniel E. Krutz, Nuthan Munaiah, Anthony Peruma, and Mohamed Wiem Mkaouer. 2017. Who Added That Permission to My App? An Analysis of Developer Permission Changes in Open Source Android Apps. IEEE, 165-169. https://doi.org/10.1109/MOBILESoft.2017.5

[11] Li Li. 2017. Mining androzoo: A retrospect. In Software Maintenance and Evolution (ICSME), 2017 IEEE International Conference on. IEEE, 675-680.

[12] Ivano Malavolta, Stefano Ruberto, Tommaso Soru, and Valerio Terragni. 2015. Hybrid mobile apps in the google play store: An exploratory investigation. In Proceedings of the Second ACM International Conference on Mobile Software Engineering and Systems. IEEE Press, 56-59.

[13] Maleknaz Nayebi, Homayoon Farrahi, and Guenther Ruhe. 2016. Analysis of marketed versus not-marketed mobile app releases. In Proceedings of the 4th International Workshop on Release Engineering. ACM, 1-4.

[14] Luca Pascarella, Franz-Xaver Geiger, Fabio Palomba, Dario Di Nucci, Ivano Malavolta, and Alberto Bacchelli. 2018. Self-Reported Activities of Android Developers. In 5th IEEE/ACM International Conference on Mobile Software Engineering and Systems. ACM, New York, NY, to appear.

[15] Christoffer Rosen and Emad Shihab. 2016. What are mobile developers asking about? a large scale study using stack overflow. Empirical Software Engineering 21, 3 (2016), 1192-1223.

[16] Anthony I Wasserman. 2010. Software engineering issues for mobile application development. In Proceedings of the FSE/SDP workshop on Future of software engineering research. ACM, 397-400. 